\title{
Non-Hermitian matter-wave mixing in Bose-Einstein condensates: Dissipation-induced amplification
}

\author{
S. Wüster ${ }^{1,2, *}$ and R. El-Ganainy ${ }^{3, \dagger}$ \\ ${ }^{1}$ Department of Physics, Bilkent University, Ankara 06800, Turkey \\ ${ }^{2}$ Department of Physics, Indian Institute of Science Education and Research, Bhopal, Madhya Pradesh 462 023, India \\ ${ }^{3}$ Department of Physics and Henes Center for Quantum Phenomena, Michigan Technological University, Houghton, Michigan 49931, USA
}

(Received 17 October 2016; revised manuscript received 5 June 2017; published 6 July 2017)

\begin{abstract}
We investigate the nonlinear scattering dynamics in interacting atomic Bose-Einstein condensates under non-Hermitian dissipative conditions. We show that, by carefully engineering a momentum-dependent atomic loss profile, one can achieve matter-wave amplification through four-wave mixing in a quasi-one-dimensional nearly-free-space setup - a process that is forbidden in the counterpart Hermitian systems due to energy mismatch. Additionally, we show that similar effects lead to rich nonlinear dynamics in higher dimensions. Finally, we propose a physical realization for selectively tailoring the momentum-dependent atomic dissipation. Our strategy is based on a two-step process: (i) exciting atoms to narrow Rydberg or metastable excited states, and (ii) introducing loss through recoil; all while leaving the bulk condensate intact due to protection by quantum interference.
\end{abstract}

DOI: 10.1103/PhysRevA.96.013605

\section{INTRODUCTION}

Wave mixing is a fundamental process associated with nonlinear interactions that involve several wave components. The most common wave mixing processes involve three and four interacting waves. In optics, both of these processes occur [1] and are utilized in many applications ranging from second-harmonic generation and parametric amplification to the generation of entangled photons and squeezed light. In atomic Bose-Einstein condensates (BECs), nonlinear interactions naturally lead to four-wave mixing (4WM) [2-5]-a feature that is employed to generate entangled atomic beams [6-8]. An efficient wave mixing- or scattering process must satisfy energy and momentum conservation. This poses severe limitations for one-dimensional systems, which necessitate dispersion engineering in optics and prohibit wave mixing in homogeneous quasi-one-dimensional (quasi-1D) BEC setups [9], as illustrated in Fig. 1(a).

Inspired by recent activities on nonlinear $\mathcal{P} \mathcal{T}$-symmetric photonic structures [10-22] particularly on non-Hermitian optical parametric amplification [20], we show here that nonHermitian engineering in BECs can alleviate some of these limitations and enable a host of intriguing effects. In particular, we show that, in a $4 \mathrm{WM}$ scattering process with degenerate input states and two distinct output modes, the introduction of a selective atomic loss in just one of the output modes can lead to the amplification of the second output state. The physical mechanism underlying this counterintuitive effect can be understood by recalling the damped harmonic oscillator: loss or damping leads to a finite frequency width around the resonance and thus allows for additional flexibility in the fulfillment of energy and momentum conservation (see Fig. 1). Interestingly, this strategy opens up 4WM channels in quasi1D BEC setups, which otherwise exist only through dispersion

\footnotetext{
*sebastian@iiserb.ac.in

†ganainy@mtu.edu
}

engineering via optical lattices $[9,23,24]$ or by invoking internal degrees of freedom to fulfill energy conservation [25].

As we will show later, the required momentum-dependent atom loss can be engineered by coupling the condensate atoms to rapidly decaying electronic states, allowing these atoms to be ejected from the trap via photon recoil. Momentum selectivity enters via the Doppler effect in a similar fashion as in laser cooling. To implement these features, we adapt a cooling scheme [26-28] based on electromagnetically induced transparency (EIT) [29] by exchanging low-lying excited states with energetically narrow highly excited Rydberg states [30,31] or metastable states [32,33]. Their small natural linewidths are required to resolve the small atomic momenta and hence Doppler shifts in the BEC, while EIT quantum interference protects the bulk condensate at zero velocity from loss.

As our main result, we demonstrate that certain loss profiles $\gamma(\boldsymbol{k})$ can lead to the amplification of a phonon wave packet in homogeneous quasi-1D condensates through wave mixing. Afterwards, we investigate the collisions of three separate condensates [4] under non-Hermitian conditions and demonstrate features associated with loss-induced scattering channels. Our results open up a host of scenarios for atomic wave mixing experiments and boost BECs as a platform for the study of non-Hermitian physics [34,35] with a diverse pool of loss processes to generate non-Hermiticity. Additionally, they may enhance the utility of BECs for atom interferometry [36], atom lasers [37,38], or entanglement generation [39-42]. Finally, we provide a specific mechanism of how the required loss profiles can be experimentally implemented.

The article is organized as follows: In Sec. II we present the essential mechanism of four-wave mixing in the presence of loss, with full details of the calculation in Appendixes A and B. Analytical formulas are numerically validated in Appendix C. In Sec. III we demonstrate wave mixing processes amplified through atomic loss in a one-dimensional scenario, moving to two dimensions in Sec. IV. All effects are shown to be robust against quantum and thermal fluctuations by using the truncated Wigner approximation described briefly 


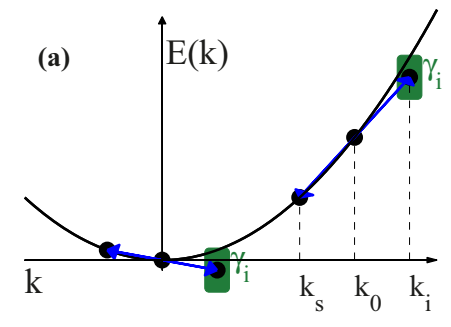

(b)

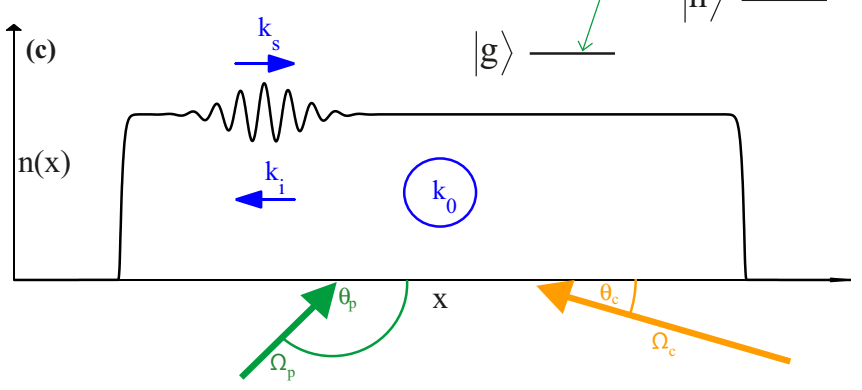

FIG. 1. (a) The nonlinear dispersion relation $E(k)=\hbar^{2} k^{2} /(2 m)$ of matter waves usually prohibits one-dimensional scattering processes of the kind $2 k_{0} \rightarrow k_{s}+k_{i}$, as shown. This can be remedied by engineering a loss-induced width $\gamma_{i}$ of the final scattering state (green). (b) Internal $\Lambda$-type level scheme of the atoms used for engineering the loss, which we discuss in Sec. V. $|g, h\rangle$ are long-lived ground states, $|r\rangle$ may be a Rydberg state with decay rate $\gamma$. Green and yellow lines and symbols represent two laser beams, the detuning of which depends on atomic velocity $\mathbf{v}$ through the Doppler shift. (c) Matter-wave packet (signal) of center wave number $k_{s}$ within a quasi-1D BEC (pump) at rest $\left(k_{0}=0\right)$. Laser-induced loss only for wave numbers $\left(\sim\right.$ momenta) near $k_{i}=-k_{s}$ (idler) cause the amplification of the signal through slight depletion of the pump. $\theta_{p}$ and $\theta_{c}$ are the angles of laser beams in panel (b) with the long axis of the condensate.

in Appendix E. Since the results of the earlier sections do not rely on how exactly momentum-dependent loss is realized, we defer our proposal for a practical implementation of it to Sec. V, with some more details in Appendix D.

\section{NON-HERMITIAN FOUR-WAVE MIXING}

We start by assuming a quasi-1D BEC (see, e.g., [43-45]) made of atoms with mass $m$. Within the mean-field GrossPitaevskii equation (GPE), the system can be described in the momentum representation:

$$
\begin{aligned}
i \hbar \frac{\partial \phi(k)}{\partial t}= & \frac{\hbar^{2} k^{2}}{2 m} \phi(k)+U \int_{-\infty}^{\infty} d k_{1} \int_{-\infty}^{\infty} d k_{2} \int_{-\infty}^{\infty} d k_{3} \\
& \times \delta\left(k_{2}+k_{3}-k_{1}-k\right) \phi^{*}\left(k_{1}\right) \phi\left(k_{2}\right) \phi\left(k_{3}\right) \\
& -i \hbar \gamma(k) \phi(k),
\end{aligned}
$$

where $\delta(k)$ is a Dirac $\delta$ function expressing the conservation of momentum during atomic ( $s$-wave) scattering processes with interaction constant $U$. We also include momentum-dependent loss, with a rate $2 \gamma(k)$ per atom. The components of the condensate momentum space wave function $\phi(k)$ at different momenta $k_{n}$ can couple through atomic scattering processes, possibly giving rise to wave mixing.
To first gain insight into the effect of loss on wave mixing processes, we consider a simplification of the nonlinear manymode problem (1), where only three discrete momenta $k_{0}, k_{s}, k_{i}$ are involved in a scattering process, as shown in Fig. 1. These are referred to as pump, signal, and idler modes, respectively, in analogy to the optical scenario [20]. Furthermore, we assume that only atoms in mode $k_{i}$ experience losses with rate $\gamma=\gamma\left(k_{i}\right)$. Next, we express the momentum amplitudes in an interaction picture, $\Phi_{n}=\phi\left(k_{n}\right) \sqrt{\Delta k} \exp \left[i\left(E_{n}+\epsilon_{n}\right) t / \hbar\right]$, with $E_{n}=\hbar^{2} k_{n}^{2} /(2 m)$ and $\epsilon_{n}=\{0, \hbar \kappa / 2,-\hbar \kappa / 2\}$ for $n \in$ $\{0, s, i\}$. Here $\Delta k$ is a wave-number scaling factor, chosen as the width of the discrete momentum mode at $k_{n}$, and $\kappa=$ $(\Delta E-U \rho) / \hbar$, with energy mismatch $\Delta E=E_{s}+E_{i}-2 E_{0}$, for a homogeneous condensate with density $\rho$. Considering an isolated wave mixing process involving only $k_{0, s, i}$ rather than more general complex many-mode interactions as in Eq. (1) is justified under the condition $\Delta E \gg U \rho$. In the complementary case of $\Delta E \sim U \rho$, the interaction energy itself can usually enable a much larger set of scattering channels, leading to a many-mode problem.

It is now straightforward to show, as we do in Appendix B, that as long as the bulk BEC at $k_{0}$ remains largely unaffected (undepleted pump approximation), we have

$$
\begin{aligned}
i \hbar \frac{\partial \Phi_{s}}{\partial t} & =\left(2 U \rho+\frac{\hbar \kappa}{2}\right) \Phi_{s}+U \rho \Phi_{i}^{*}, \\
i \hbar \frac{\partial \Phi_{i}}{\partial t} & =-\left(2 U \rho+\frac{\hbar \kappa}{2}\right) \Phi_{i}-U \rho \Phi_{s}^{*}-i \hbar \gamma \Phi_{i} .
\end{aligned}
$$

Equation (B5) admits solutions of the form $\boldsymbol{\Phi}(t)=\boldsymbol{\Phi}^{(+)}$ $\exp \left[-i \lambda^{(+)} t\right]+\boldsymbol{\Phi}^{(-)} \exp \left[-i \lambda^{(-)} t\right]$, where $\boldsymbol{\Phi}(t)=\left[\Phi_{s}, \Phi_{i}\right]^{T}$ and the eigenvalues

$$
\begin{aligned}
\lambda^{( \pm)}= & -i \frac{\gamma}{2} \pm \frac{1}{2}\left[-\gamma^{2}+4(2 U \rho / \hbar+\kappa / 2)^{2}\right. \\
& \left.-4(U \rho / \hbar)^{2}+4 i \gamma(2 U \rho / \hbar+\kappa / 2)\right]^{1 / 2}
\end{aligned}
$$

In order for amplification to take place, at least one of the above eigenvalues must satisfy $\operatorname{Im}\left[\lambda^{( \pm)}\right]>0$. Under the condition $\Delta E \gg U \rho$, we find the imaginary part of the amplifying eigenvalue:

$$
\operatorname{Im}\left[\lambda^{(+)}\right]=\frac{(U \rho / \hbar)^{2} \gamma}{\gamma^{2}+(\Delta E / \hbar)^{2}} .
$$

From Eq. (4), one can make the following important observations: (i) $\operatorname{Im}\left[\lambda^{(+)}\right]$is maximal when $\gamma \approx \Delta E / \hbar$. Intuitively, the loss then broadens the energetic width of the idler state just enough to satisfy energy conservation, as shown in Fig. 1 . (ii) The condition $\Delta E \gg U \rho$ implies $\operatorname{Im}\left[\lambda^{(+)}\right] \ll U \rho / \hbar$. Since $U \rho / \hbar$ sets the timescale for nonlinear BEC mean-field dynamics, the amplification will take place at a slower pace than the interaction dynamics. We refer to Appendix $\mathrm{C}$ for a numerical validation of the discussion so far.

\section{MATTER-WAVE SIGNAL AMPLIFICATION}

We proceed to demonstrate the nonlinear amplification of a matter wave, enabled by dissipation, in a finite-size multimode system. We consider an approximately homogeneous, quasione-dimensional, ${ }^{87} \mathrm{Rb}$ BEC in a box trap, such as, e.g., in the experiment described in Ref. [46], with length $L=640 \mu \mathrm{m}$ 

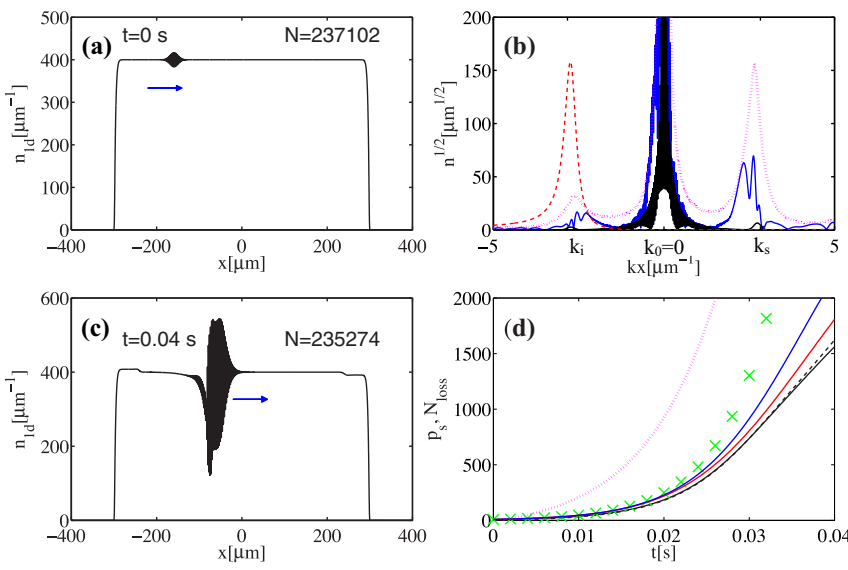

FIG. 2. 1D matter-wave parametric amplification. (a) Initially a small wave packet on a large top-hat-shaped condensate wave packet is traveling near wave number $k_{s}$ to the right. (b) Momentum spectra of condensate at $t=0$ (black) and $t=0.04 \mathrm{~s}$ (blue) as well as loss spectrum $\gamma(k)$ in arbitrary units (red dashed). See Sec. V for a derivation of the lineshape. We also plot the momentum spectrum at $t=0.04 \mathrm{~s}$ in the presence of quantum noise (magenta dotted). (c) After $0.04 \mathrm{~s}$ action of loss on the wave number $k_{i}=-k_{s}$, the signal has been significantly amplified, with intensity drawn from the background (pump) condensate near $k_{0} \approx 0$. (d) The signal strength $p_{s}=\int_{k_{s}-K}^{k_{s}+K}|\phi(k)|^{2} d k$ from the GPE (solid black) increases in exact correspondence to the number of lost atoms $N_{\text {loss }}=N(0)-N(t)$ (dashed black). We also show the corresponding TWA signal $p_{s}=$ $\int_{k_{s}-K}^{k_{s}+K} n_{\text {tot }}(k) d k$ (magenta dotted). Suitably chosen [49] simulations for a homogeneous condensate give similar results: Eq. (6) of the Supplemental Material (solid blue) and Eq. (1) (solid red). Finally we also show the analytical solution of Eq. (B5) using Eq. (3) $(x$ green). See also the movie in the Supplemental Material. Beyond the parameters stated in the text or clear from the figure, we have used a phonon wave packet with $A_{s}=600 \mathrm{~m}^{-1 / 2}, \sigma=9.6 \mu \mathrm{m}$. The loss spectrum $\gamma(k)$, see Sec. V, is based on $\Gamma=(2 \pi) / \tau$ with $\tau=3.6 \mu \mathrm{s}$ (corresponding to $|r\rangle=\left|15, p_{1 / 2}\right\rangle[50]$ ), $\Omega_{p}=(2 \pi) 12$ $\mathrm{kHz}, \Omega_{c}=(2 \pi) 395 \mathrm{kHz}, \Delta_{0}=(2 \pi) 3 \mathrm{MHz}$ at the laser wavelength $\lambda=308 \mathrm{~nm}$ and $a_{p}=1, a_{c}=-1$.

and transverse trapping frequency $\omega_{\perp}=(2 \pi) 100 \mathrm{~Hz}$. The system is sketched in Fig. 1(a). A one-dimensional choice simplifies the numerical solution of Eq. (1), for which we use XMDS $[47,48]$, but more importantly represents a regime where, in the absence of loss, matter-wave mixing processes are suppressed in one dimension. Starting from the ground state of the condensate in the box trap, we imprint a small "signal" matter wave packet, $\phi(x) \rightarrow \phi(x)+A_{s} \exp [-(x-$ $\left.\left.x_{0}\right)^{2} /(2 \sigma)+i k_{s} x\right]$, with amplitude $A_{s}$ and width $\sigma$ centered around $k_{s}=2.7 \mu \mathrm{m}^{-1}$ onto the BEC, as shown in Fig. 2(a). The thick part of the line are unresolved fast oscillations with wavelength $\lambda_{s}=2 \pi / k_{s}$.

Figure 2(b) depicts the initial wave packet of Fig. 2(a) in Fourier space, where we distinguish the sinc-shaped bulk condensate peak ("pump," $k_{0}$ ) and the small signal near $k_{s}$. We now assume that the loss is switched on at time $t=0$, with profile $\gamma(\boldsymbol{k})$ shown as red dashed curve in Fig. 2(b). Note that the spectral distribution of the loss is centered around $k_{i}=-k_{s}$. We discuss later how to obtain such a profile. Action of this loss results in a significant amplification of the matterwave signal, as shown in Fig. 2(c), together with visible bulk condensate depletion behind the passing signal wave packet. In momentum space this manifests as rapid growth of population in momentum modes around $k_{s}$ as shown in Fig. 2(d). At later times, the amplification triggers complicated nonlinear multimode dynamics, as we can see also the movie in the Supplemental Material [51]. We note that, in the absence of dissipation, none of these effects would take place and instead the initial wave packet of Fig. 2(a) would bounce off the box edges without any change in its amplitude.

For comparison, we added to Fig. 2(d) the signal mode growth rate predicted by Eq. (3) for a homogeneous condensate closely matching the present scenario in the three-mode approximation $(x$ green), which agrees well with full numerical solutions. Equations (3) and (4) can thus provide useful guidance towards the parameters supporting non-Hermitian signal amplification. We have repeated our simulations including quantum corrections beyond the GPE by using the truncated Wigner method [52-59]; see Appendix E. As demonstrated in Fig. 2 the effect is robust, the increased seed amplitude through quantum noise even leads to a larger signal (but same amplification factor).

We emphasize that, while no significant dynamics takes place without the loss, the small fraction of lost atoms $(\sim 1 \%)$ enables a dramatic change in matter-wave dynamics.

\section{NONDEGENERATE QUASI-TWO-DIMENSIONAL CASE}

The scenario discussed above represents degenerate fourwave mixing, where two of the initial momenta of a scattering process coincide $\left(k_{0}\right)$. A more general four-wave mixing process involves four different momentum components and has been exploited for condensate collisions [60,61], which lay the basis for studying Einstein-Podolsky-Rosen (EPR) correlations with massive particles $[39,62,63]$. In all these processes, conservation of energy and momentum plays a crucial role. A striking demonstration of this is the Bose stimulated creation of a new momentum component after the collision of three different condensates having distinct initial momenta [4].

To illustrate how engineering the matter dissipation can seemingly relax energy-momentum constraints, we consider a two-dimensional (2D) scattering process. Figure 3(a) depicts three superimposed condensate clouds, with $N_{0}=60000$ atoms each, in a pancake trap with $\omega_{\perp}=(2 \pi) 200 \mathrm{~Hz}$. Their initial velocities are $\left|v_{0,2}\right|=0.4 \mathrm{~mm} / \mathrm{s}$, and $\left|v_{1}\right|=0.66 \mathrm{~mm} / \mathrm{s}$, as indicated. Trapping in the $x, y$ directions is neglected. Since, e.g., the momentum-allowed scattering process $p_{0}+$ $p_{2} \rightarrow p_{1}+p_{3}$ with $p_{3}=-p_{1}$ violates energy conservation, the clouds pass each other in the absence of loss, with just diffusive- and interaction-induced broadening. However, adding momentum-dependent loss with a peak within the white stripes of Fig. 3(c) (i.e., around $p_{3}$ ) introduces an energy width alleviating these constraints, as discussed earlier. Consequently, our simulations show a $15 \%$ increase of the signal around $p_{1}$ during the condensate interaction, originating from these stimulated scattering events. Even if quantum fluctuation at the level of $0.4 \%$ of the atomic peak density are added within the truncated Wigner approximation (TWA) $[1,51]$, the amplification qualitatively persists with quantitative deviations. All results should be experimentally accessible. 

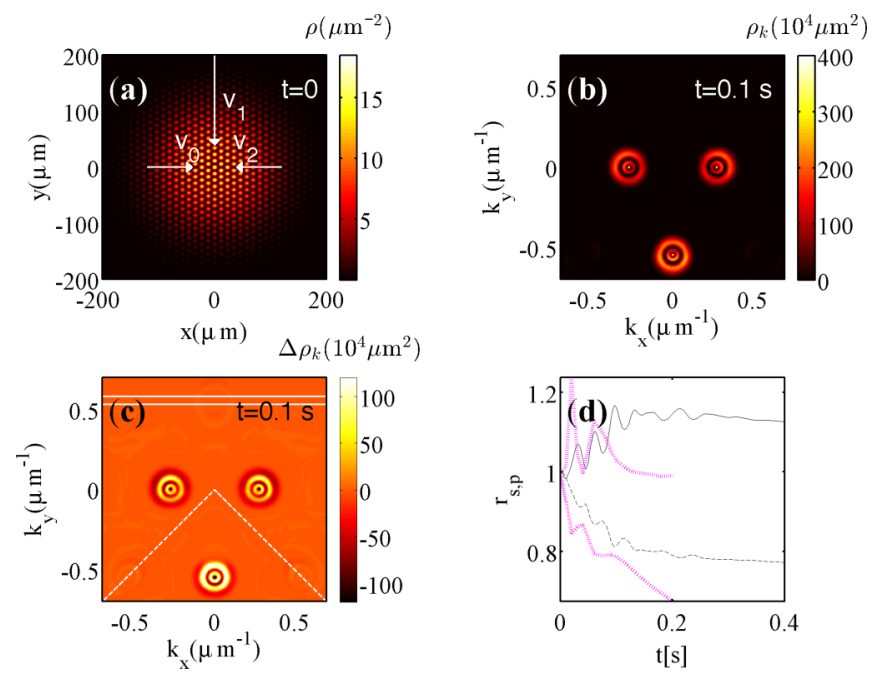

FIG. 3. Non-Hermitian effects in $2 \mathrm{D}$ wave mixing. (a) Initial condensate density $\rho=|\phi(x, y)|^{2}$, showing interference due to multiple superimposed velocity components (white arrows). (b) Momentum space density $\rho_{k}=\left|\phi\left(k_{x}, k_{y}\right)\right|^{2}$ after $0.1 \mathrm{~s}$ of wave mixing in the presence of loss. (c) Difference $\Delta \rho_{k}=\rho_{k}-\rho_{k 0}$, where $\rho_{k 0}$ corresponds to the case without loss. The white horizontal lines indicate the FWHM of a loss peak shaped as that in Fig. 2(c) in the $y$ direction and independent of $x$. White dashed lines separate our signal bins, as discussed in the text. (d) Signal- and pump population ratios (see text) from GPE (solid and dashed black, respectively) and TWA (matching magenta dashed). See also the movie in the Supplementary Material. For this simulation, the loss spectrum $\gamma(k)$ was adjusted to $\Omega_{p}=(2 \pi) 3 \mathrm{kHz}, \Omega_{c}=(2 \pi) 205 \mathrm{kHz}, \Delta_{0}=(2 \pi) 4 \mathrm{MHz}$ compared with Fig. 2.

\section{ENGINEERING MOMENTUM SELECTIVE LOSS IN A BOSE-EINSTEIN CONDENSATE}

It was crucial for the development so far that the loss term $\gamma(k)$ affects only atoms in the idler mode $k_{i}$ and not $k_{0 / s}$. Here we discuss one possible method to reach that goal, exploiting Doppler-shift techniques borrowed from laser cooling, which also hinges on velocity-selective manipulations of atoms.

There are two additional challenges here: the comparatively small velocities involved in BEC dynamics and avoiding excess loss of the majority of condensate atoms. Both can be met by employing a laser-cooling technique that involves quantum interference through EIT, as has been proposed $[26,27]$ and demonstrated [28] for the cooling of ions.

In this scheme, condensate atoms in their ground state $|g\rangle$ are coupled to two laser beams, in the off-resonant $\Lambda$ configuration shown in Fig. 1(b) of the main article. The corresponding Hamiltonian for one atom in the rotating-wave approximation reads

$$
\begin{aligned}
\hat{H}_{\mathrm{EIT}}= & {\left[-\frac{\Omega_{\mathrm{p}}}{2} \hat{\sigma}_{r g}-\frac{\Omega_{\mathrm{c}}}{2} \hat{\sigma}_{r h}+\right.\text { H.c. }} \\
& \left.+\Delta_{p}(\boldsymbol{v}) \hat{\sigma}_{r r}+\left[\Delta_{p}(\boldsymbol{v})-\Delta_{c}(\boldsymbol{v})\right] \hat{\sigma}_{h h}\right]
\end{aligned}
$$

with $\hat{\sigma}_{k k^{\prime}}=\left[|k\rangle\left\langle k^{\prime}\right|\right]$. The detunings $\Delta_{p}$ and $\Delta_{c}$ are implicitly dependent on the velocity of the atom $\boldsymbol{v}$ through the Doppler shift $\Delta_{p}=\Delta_{0}-\boldsymbol{v} \cdot \boldsymbol{q}_{p}$ and $\Delta_{c}=\Delta_{0}-\boldsymbol{v} \cdot \boldsymbol{q}_{c}$, where $\Delta_{0}$ is a common base detuning and $\boldsymbol{q}_{p}$ and $\boldsymbol{q}_{c}$ are the respective laser wave vectors. For our one-dimensional simulation of Fig. 2, we in practice write this as $\boldsymbol{v} \cdot \boldsymbol{q}_{p}=v|\boldsymbol{q}| a_{p}$ and $\boldsymbol{v} \cdot \boldsymbol{q}_{c}=v|\boldsymbol{q}| a_{c}$, where $v$ is the one-dimensional velocity, $|\boldsymbol{q}| \approx\left|\boldsymbol{q}_{p}\right| \approx\left|\boldsymbol{q}_{c}\right|$ is the laser wave number and $a_{p}=\cos \theta_{p}$ and $a_{c}=\cos \theta_{c}$ account for the angle between the probe and coupling beams and our one-dimensional condensate. The latter are sketched in Fig. 1(c) and afford additional flexibility in the design.

The system evolves according to the Lindblad master equation for the density matrix $\hat{\rho}(h=1)$ :

$$
\dot{\hat{\rho}}=-i[\hat{H}, \hat{\rho}]+\sum_{\alpha} \mathcal{L}_{\hat{L}_{\alpha}}[\hat{\rho}],
$$

where the superoperators $\mathcal{L}_{\hat{L}_{\alpha}}[\hat{\rho}]$, with $\mathcal{L}_{\hat{O}}[\hat{\rho}]=\hat{O} \hat{\rho} \hat{O}^{\dagger}-$ $\left(\hat{O}^{\dagger} \hat{O} \hat{\rho}+\hat{\rho} \hat{O}^{\dagger} \hat{O}\right) / 2$ describe spontaneous decay of the excited state $|r\rangle$ to either of the ground states via $\hat{L}_{\alpha=1}=$ $\sqrt{\Gamma / 2} \hat{\sigma}_{g r}$ and $\hat{L}_{\alpha=2}=\sqrt{\Gamma / 2} \hat{\sigma}_{h r}$.

As usual, the timescale of atom-light coupling is much faster than BEC dynamics so that we can assume the atoms to settle into a steady state $\hat{\rho}^{(\infty)}$ with $\dot{\hat{\rho}}=0$, dependent on the atomic velocity through the Doppler shift.

In contrast to laser-cooling, we now crucially assume that the photon recoil energy $E_{r}$ of decayed atoms is larger than the trap depth - a condition that can be fulfilled experimentally. Consequently, an atom emitting a photon will be ejected out of the trap and is considered lost. Loss is thus linked to spontaneous decay, and we obtain the velocity-dependent (wave-number-dependent) loss as $\gamma(k)=\Gamma\left\langle r\left|\hat{\rho}^{(\infty)}\right| r\right\rangle / 2$.

Let us consider the situation without the atomic state $|h\rangle$ first: Setting $\Omega_{c}=0$ and $\Delta_{c}=0$, Fig. 1(b) reduces to a two-level system and $\rho_{r r}=\left\langle r\left|\hat{\rho}^{(\infty)}\right| r\right\rangle$ takes the well-known Lorentzian spectral lineshape. For our scheme to function as intended, the width $\Delta k$ of $\rho_{r r}$ must be tailored such that the atomic loss is significant only for the idler matter waves $k_{i}$, but negligible on the bulk background condensate $k_{0}$. This is challenging since the Doppler shifts caused by velocities such as used in Fig. 2 are as small as $\delta \omega=v_{s} q_{p} \approx 40 \mathrm{kHz}$. We are thus led to the use of "long-lived" excited states for $|r\rangle$, such as Rydberg states [30,31] or metastable spin triplets in two-electron atoms [32,33] which have linewidths of this magnitude. The ensuing combination of Rydberg and BEC physics hold promise as an exciting emerging discipline [64-70].

However, even for these states, the strong tails of the Lorentzian line can cause a significant loss of bulk condensate atoms near $k_{0} \approx 0$. It is in order to overcome this obstacle that the excitation scheme includes coupling $|r\rangle$ to another hyperfine ground state $|h\rangle$. The resulting $\Lambda$-type level scheme enables a complete suppression of non-Doppler-shifted excitation (loss) at $k \approx 0$ via quantum interference effects (EIT). While matter loss at the signal momenta $k_{s}$ cannot be fully suppressed in this scheme, it can be made sufficiently small.

If we evaluate the modified loss $\gamma(k)=\Gamma\left\langle r\left|\hat{\rho}^{(\infty)}\right| r\right\rangle / 2$ in the presence of the state $|h\rangle$, we obtain

$$
\begin{aligned}
\gamma(k)= & 4 \Delta_{\text {eff }}^{2} \Omega_{p}^{2} \Omega_{c}^{2} /\left\{\left[16\left(\Delta_{c}^{2}+\Omega_{c}^{2}\right) \Delta_{\text {eff }}^{2}+3 \Omega_{c}^{4}\right] \Omega_{p}^{2}\right. \\
& +\left(3 \Omega_{c}^{2}-8 \Delta_{c} \Delta_{\text {eff }}\right) \Omega_{p}^{4}+\Omega_{p}^{6}+4 \Gamma^{2} \Delta_{\text {eff }}^{2}\left(\Omega_{p}^{2}+\Omega_{c}^{2}\right) \\
& \left.+\left(4 \Delta_{\text {eff }} \Delta_{p} \Omega_{c}+\Omega_{c}^{3}\right)^{2}\right\}
\end{aligned}
$$

with $\Delta_{\text {eff }}=\Delta_{p}-\Delta_{c}$. 

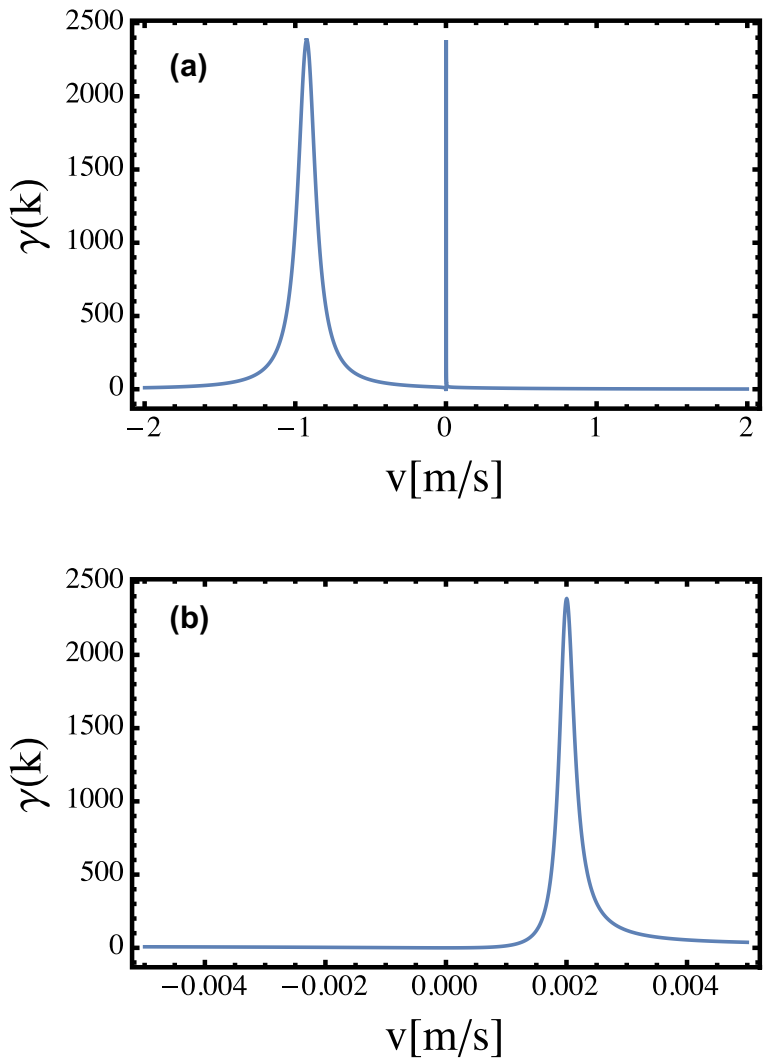

FIG. 4. (a) Loss spectrum engineered through $\Lambda$ scheme as a function of atomic velocity, for parameters as in Fig. 2 of the main article. (b) The same, but zoomed onto the narrow resonance feature near $v=v_{s}=0.002 \mathrm{~m} / \mathrm{s}$.

We show the atomic excitation spectrum, or loss spectrum (7), as a function of velocity in Fig. 4. On the wider velocity range in Fig. 4(a) we see a broad and a narrow resonance feature, corresponding to the two eigenstates of the strongly coupled $|r\rangle,|h\rangle$ subspace. Our interest is in the narrow spectral feature, zoomed upon in Fig. 4(b), which realizes the velocity width required for the proposal. The state is narrow due to the small probability to be in the decaying $|r\rangle$ level. This spectral feature gives rise to the loss spectrum also shown in Fig. 2.

Note that, in our argument we essentially rely on a hierarchy of three timescales: $\tau \ll T_{\mathrm{rec}} \ll T_{\mathrm{BEC}}$, where the excited state lifetime $\tau=\gamma^{-1}$ sets the scale for radiative decay establishing an atomic steady state, the recoil time $T_{\text {rec }}$ determines how fast a decaying atom is lost from the trap, and $T_{\mathrm{BEC}}$ denotes the timescale of condensate dynamics of interest. We estimate $T_{\text {rec }}=a_{\perp} / v_{\text {rec }}$, where $a_{\perp}=\sqrt{\hbar / m / \omega_{\perp}}$ is the radial trapping width and $v_{\text {rec }}=2 \hbar\left|q_{p}\right| / m$ is the recoil velocity.

For the parameters of Fig. 2 in the main article we have $\tau=3.6 \times 10^{-6} \mu \mathrm{s}, T_{\mathrm{rec}}=36 \mu \mathrm{s}$, and $T_{\mathrm{BEC}} \sim 1 \mathrm{~ms}$, fulfilling the hierarchy.

In addition to the mechanism of atom loss described above, the laser beams will also cause a dispersive energy shift $\delta E(k)$, as discussed in Appendix D. Although this contribution can be made small enough to have a minor effect on condensate dynamics, it is not entirely negligible. We thus included it in the simulations above as a modification of atomic dispersion $\sim \delta E(k) \phi(k)$ on the right-hand side of Eq. (1). While it does slightly affect BEC dynamics, the amplification phenomena discussed are entirely due to the dissipative contribution.

\section{CONCLUSION AND OUTLOOK}

We have proposed a mechanism based on spectrally engineered matter dissipation to control the nonlinear scattering dynamics in BEC systems. More specifically, we have demonstrated that, by introducing a momentum-dependent loss profile, scattering processes in certain directions can be enhanced. When applied to quasi-1D BECs, our strategy enables efficient wave mixing in regimes that would have been inaccessible under Hermitian conditions. Similarly, we have demonstrated that spectrally engineered dissipation can be used to open new scattering channels in quasi-2D setups.

We emphasize that our primary results; namely, efficient four-wave mixing via matter loss, are valid in general and not pertinent to the examples studied here. Also alternative practical realizations of loss profiles $\gamma(\boldsymbol{k})$ would yield the same results.

The fundamental effects presented in our work further suggest BEC as an accessible laboratory platform for the study of non-Hermitian quantum mechanics, where interesting new features might arise from inelastic nonlinear loss processes in condensates, such as two- and three-body losses [55,71-73]. The new wave-mixing channels may also benefit engineering quantum-atom-optical devices, such as interferometers or entangled-atom sources.

\section{ACKNOWLEDGMENT}

R.E. acknowledges support from the Henes Center for Quantum Phenomena at Michigan Technological University.

\section{APPENDIX A: NON-HERMITIAN FOUR-WAVE MIXING}

This section presents a detailed derivation of the analytical treatment of wave mixing in the presence of losses, leading to Eq. (4) in Sec. II.

For completeness, we begin with the position-space GPE of a 1D BEC in a transversely tight trap by considering $N=$ $\int d x|\Psi(x)|^{2}$ atoms of mass $m$ in one spatial dimension and within an external trapping potential $V(x)$ :

$$
i \hbar \frac{\partial \Psi(t, x)}{\partial t}=\left[-\frac{\hbar^{2}}{2 m} \frac{\partial^{2}}{\partial x^{2}}+V(x)+U|\Psi(t, x)|^{2}\right] \Psi(t, x) .
$$

Here $U$ is an effective 1D nonlinear coefficient, containing, e.g., properties of tight transverse trapping. It can be calculated from the three-dimensional (3D) interaction constant $U_{3 \mathrm{D}}=$ $4 \pi \hbar^{2} a_{s} / m$, where $a_{s}$ is the $s$-wave scattering length, via $U=$ $U_{3 \mathrm{D}} /\left(2 \pi \sigma_{\perp}^{2}\right)$, where $\sigma_{\perp}$ is the width of the harmonic-oscillator ground state in the tightly trapped transverse direction $\sigma_{\perp}=$ $\sqrt{\hbar /\left(m \omega_{\perp}\right)}$, and $\omega_{\perp}$ is the trapping frequency of the transverse trap.

To facilitate our analysis, it is more instructive to write Eq. (A1) in the momentum representation after neglecting the potential term $V(x)$. We use 


$$
\begin{aligned}
& \Psi(t, x)=\int d k \exp [i k x] \bar{\phi}(t, k) / \sqrt{2 \pi} \text { to obtain } \\
& \begin{aligned}
i \hbar \frac{\partial \bar{\phi}(t, k)}{\partial t}= & \frac{\hbar^{2} k^{2}}{2 m} \bar{\phi}(t, k)+\frac{U}{2 \pi} \int_{-\infty}^{\infty} d k_{1} \int_{-\infty}^{\infty} d k_{2} \int_{-\infty}^{\infty} d k_{3} \\
& \times \delta\left(k_{2}+k_{3}-k_{1}-k\right) \bar{\phi}^{*}\left(t, k_{1}\right) \bar{\phi}\left(t, k_{2}\right) \bar{\phi}\left(t, k_{3}\right),
\end{aligned}
\end{aligned}
$$

where $\delta(k)$ is a Dirac $\delta$ function expressing the conservation of momentum during atomic-scattering processes.

In principle, Eq. (A2) contains complete information about the system. However, to gain more insight into a specific wavemixing process such as those represented in Fig. 1(a), we proceed by isolating a few specific momentum modes. We do this by adopting a discrete representation of Eq. (A2). This is done by using $\int d k \rightarrow \sum \Delta k$ and $\bar{\phi}\left(t, k_{n}\right)=\bar{\phi}_{n} / \sqrt{\Delta k}$ (we drop the explicit reference to the time variable $t$ ), to obtain

$$
i \hbar \frac{\partial \bar{\phi}_{p}}{\partial t}=\frac{\hbar^{2} k_{p}^{2}}{2 m} \bar{\phi}_{p}+U_{d} \sum_{n, m, l} \delta_{k_{p}+k_{n}-k_{m}-k_{l}} \bar{\phi}_{n}^{*} \bar{\phi}_{m} \bar{\phi}_{l},
$$

where $U_{d}=U \Delta k /(2 \pi)$ and $\delta_{k_{n}}=\delta\left(k_{n}\right) \Delta k$. In our simulations $\Delta k$ represents the momentum grid spacing. Finally, we write Eq. (A3) in an interaction picture via the gauge transformation $\bar{\phi}_{n}=\phi_{n} \exp \left(-i E_{n} t / \hbar\right)$, with $E_{n}=\hbar^{2} k_{n}^{2} /(2 m)$, to get

$$
i \hbar \frac{\partial \phi_{p}}{\partial t}=U_{d} \sum_{n, m, l} \delta_{k_{p}+k_{n}-k_{m}-k_{l}} \phi_{n}^{*} \phi_{m} \phi_{l} e^{i\left(E_{p}+E_{n}-E_{m}-E_{l}\right) t / \hbar} .
$$

This equation neatly encapsulates momentum and energy conservation inherent in any atomic-scattering event.

\section{APPENDIX B: LOSS-INDUCED PHASE MATCHING}

Let us now assume a momentum-dependent single-body atom loss for the BEC by adding a term

$$
i \hbar \frac{\partial \phi(k)}{\partial t}=\cdots-i \hbar \gamma(k) \phi(k) \text {. }
$$

to the right-hand side of Eq. (1). After discretization this amounts to a term $i \hbar \frac{\partial \phi_{p}}{\partial t}=\cdots-i \hbar \gamma_{p} \phi_{p}$ in Eq. (A4).

We now further restrict Eq. (A4) to just three relevant modes, $k_{0}, k_{s}, k_{i}$, for loss acting on atoms with the idler wave number $k_{i}$ only and obtain

$$
\begin{aligned}
i \hbar \frac{\partial \phi_{0}}{\partial t}= & U_{d}\left\{2 \phi_{0}^{*} \phi_{s} \phi_{i} e^{-i\left(E_{i}+E_{s}-2 E_{0}\right) t / \hbar}\right. \\
& \left.+\left[\left|\phi_{0}\right|^{2}+2\left(\left|\phi_{s}\right|^{2}+\left|\phi_{i}\right|^{2}\right)\right] \phi_{0}\right\}, \\
i \hbar \frac{\partial \phi_{s}}{\partial t}= & U_{d}\left\{\phi_{i}^{*} \phi_{0}^{2} e^{i\left(E_{i}+E_{s}-2 E_{0}\right) t / \hbar}\right. \\
& \left.+\left[\left|\phi_{s}\right|^{2}+2\left(\left|\phi_{0}\right|^{2}+\left|\phi_{i}\right|^{2}\right)\right] \phi_{s}\right\}, \\
i \hbar \frac{\partial \phi_{i}}{\partial t}= & U_{d}\left\{\phi_{s}^{*} \phi_{0}^{2} e^{i\left(E_{i}+E_{s}-2 E_{0}\right) t / \hbar}\right. \\
& \left.+\left[\left|\phi_{i}\right|^{2}+2\left(\left|\phi_{0}\right|^{2}+\left|\phi_{s}\right|^{2}\right)\right] \phi_{i}\right\}-i \hbar \gamma \phi_{i} .
\end{aligned}
$$

\section{Analytical solution}

If the majority of condensate atoms occupy the pump mode $k_{0}$, i.e., $\left|\phi_{0}\right| \gg\left|\phi_{s}\right|,\left|\phi_{i}\right|$ (a condition equivalent to the undepleted-pump approximation in the context of nonlinear optics), the evolution equation for $\phi_{0}$ becomes

$$
i \hbar \frac{\partial \phi_{0}}{\partial t}=U_{d}\left|\phi_{0}\right|^{2} \phi_{0}
$$

with the solution $\phi_{0}(t)=\sqrt{n_{0}} e^{-i U_{d} n_{0} t / \hbar}$, where $n_{0}=\left|\phi_{0}\right|^{2}$. Here $n_{0}$ is the number of atoms in the pump mode, and $\phi_{0}$ is the pump-mode amplitude. Substitution into the remaining two equations yields

$$
\begin{aligned}
i \hbar \frac{\partial \phi_{s}}{\partial t} & =U_{d} n_{0}\left\{\phi_{i}^{*} e^{i\left(E_{i}+E_{s}-2 E_{0}-2 U_{d} n_{0}\right) t / \hbar}+2 \phi_{s}\right\}, \\
i \hbar \frac{\partial \phi_{i}}{\partial t} & =U_{d} n_{0}\left\{\phi_{s}^{*} e^{i\left(E_{i}+E_{s}-2 E_{0}-2 U_{d} n_{0}\right) t / \hbar}+2 \phi_{i}\right\}-i \hbar \gamma \phi_{i} .
\end{aligned}
$$

By introducing the quantity $\kappa=\left(E_{i}+E_{s}-2 E_{0}-2 U_{d} n_{0}\right) / \hbar$ and moving to the rotating frame $\phi_{s}=\Phi_{s} \exp (i \kappa t / 2), \phi_{i}=$ $\Phi_{i}^{*} \exp (-i \kappa t / 2)$, we obtain

$$
\begin{aligned}
& i \hbar \frac{\partial \Phi_{s}}{\partial t}=\left(2 U_{d} n_{0}+\frac{\hbar \kappa}{2}\right) \Phi_{s}+U n_{0} \Phi_{i}, \\
& i \hbar \frac{\partial \Phi_{i}}{\partial t}=-\left(2 U_{d} n_{0}+\frac{\hbar \kappa}{2}\right) \Phi_{i}-U n_{0} \Phi_{s}-i \hbar \gamma \Phi_{i} .
\end{aligned}
$$

The solution of (B5) reads

$$
\boldsymbol{\Phi}(t)=\boldsymbol{\Phi}^{(+)} \exp \left[-i \lambda^{(+)} t\right]+\boldsymbol{\Phi}^{(-)} \exp \left[-i \lambda^{(-)} t\right],
$$

with

$$
\begin{aligned}
\lambda^{( \pm)}= & -i \frac{\gamma}{2} \pm \frac{1}{2}\left[-\gamma^{2}+4\left(2 U_{d} n_{0} / \hbar+\kappa / 2\right)^{2}\right. \\
& \left.-4\left(U_{d} n_{0} / \hbar\right)^{2}+4 i \gamma\left(2 U_{d} n_{0} / \hbar+\kappa / 2\right)\right]^{1 / 2},
\end{aligned}
$$

and $\boldsymbol{\Phi}^{( \pm)}$are the eigenvectors associated with the eigenvalues $\lambda^{( \pm)}$. We have used a vector notation $\boldsymbol{\Phi}(t)=\left[\Phi_{s}, \Phi_{i}\right]^{T}$. Evidently, nonlinear amplification of atomic momentum modes in Eq. (B6) takes place if either one of the eigenvalues satisfies the condition $\operatorname{Im}\left[\lambda^{( \pm)}\right]>0$. For our present reduction of the problem to just three interacting momentum modes to be valid, we require $\Delta E \equiv E_{i}+E_{s}-2 E_{0} \gg\left(U_{d} n_{0}\right)$. Otherwise, If $\Delta E \sim\left(U_{d} n_{0}\right)$, the interaction energy itself can usually enable a much larger set of scattering channels, leading to a many-mode problem. The expression for the amplification factor then takes the form

$$
\operatorname{Im}\left[\lambda^{(+)}\right]=\frac{\left(U_{d} n_{0} / \hbar\right)^{2} \gamma}{\gamma^{2}+(\Delta \bar{E} / \hbar)^{2}}
$$

It is useful to reexpress $U n_{0}$ in terms of the homogeneous position-space density $\rho$ within a domain of size $L$. We then can write $U_{d} n_{0}=U \Delta k N_{0} /(2 \pi)=U(2 \pi / L) N_{0} /(2 \pi)=U \rho$, which is used in Eqs. (B5) and (4).

From expression (B8), we can read off the following important results: (i) $\operatorname{Im}\left[\lambda^{(+)}\right]$is maximal when $\gamma \approx \Delta E / \hbar$. In that case loss just broadens the target momentum state enough to satisfy the energy conservation relation, as shown in Fig. 1. For the parameters of Fig. 5 this predicts an optimum at $\gamma=1.2$ for which the effect is indeed much faster than the other cases shown. (ii) Since we need $\Delta E \gg U_{d} n_{0}$, we always have $\operatorname{Im}\left[\lambda^{(+)}\right] \ll \gamma$. This forces us to interpret the condition for wave mixing $\Delta E \gg U_{d} n_{0}$ as generous as possible, with 

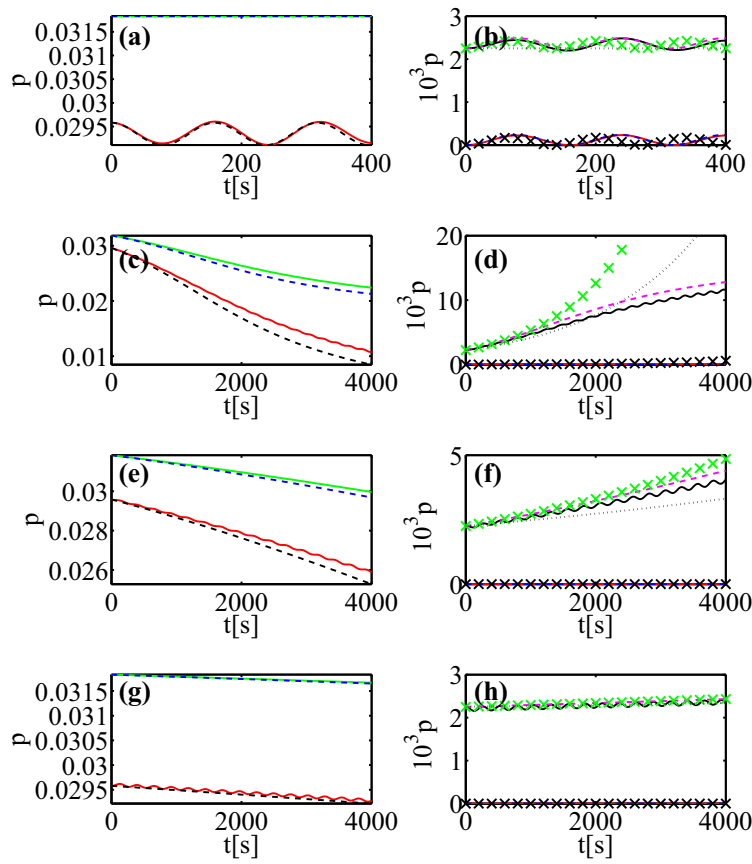

FIG. 5. Simulations of four-wave mixing in a homogenous BEC, using Eq. (1) with Eq. (B1) (solid), compared with a few-mode approximation (B2) (dashed), and the analytical solution based on Eq. (3) $(\times)$. All curves show momentum-space densities $\left|\phi\left(k_{i}\right)\right|^{2}=$ $\left|\phi_{n}\right|^{2} / \Delta k$ at various wave numbers: (right panels, upper curves) signal $k_{s}$, (right panels, lower curves) idler $k_{i}$, (left panel, lower curves) pump $k_{0}$, (left panel, upper curves) sum of all three. From top to bottom we increase the idler damping rate $\gamma$, (a), (b) $\gamma=0$, (c), (d) $\gamma=1.2$, (e), (f) $\gamma=10$, (g), (h) $\gamma=100$.

$\Delta E$ being just a few multiples of $U_{d} n_{0}$, to have the fastest possible effect. (iii) Using all these arguments together we also have $\operatorname{Im}\left[\lambda^{(+)}\right] \ll U n_{0}$. Since $U_{d} n_{0}$ sets the timescale for the nonlinear BEC dynamics, this will make the amplification effect slower than the latter.

For a case such as in Fig. 2, the last conclusion is no limitation since the bulk nonlinearity in the homogeneous case does not lead to any nontrivial evolution of the wave function. In contrast, for Fig. 3 it limits the achievable signal since, for faster amplification, nonlinearities become too large for the matter waves to pass through each other.

\section{APPENDIX C: VERIFICATION OF RESULTS}

Here we present a numerical verification of the predictions made in the previous section. To do so, we consider the case of a homogeneous BEC of $N$ atoms in periodic domain of length $L$; thus with density $\rho=N / L$. The BEC is initially given a momentum $k_{0}$ with a small fraction $A_{s}$ of the BEC seeded into the signal mode (momentum $k_{s}$ ):

$$
\Psi(x, 0)=\sqrt{\frac{N}{L}}\left(\sqrt{1-A_{s}} \exp \left[i k_{0} x\right]+\sqrt{A_{s}} \exp \left[i k_{s} x\right]\right) .
$$

The initial condition $(\mathrm{C} 1)$ is then propagated numerically under the loss spectrum $\gamma(k)=\gamma A \exp \left[-\left(k-k_{\text {loss }}\right)^{2} /\left(2 \sigma_{\text {loss }}\right)\right]$, centered around $k_{i}=2 k_{0}-k_{s}$. Other simulation parameters were chosen similar to those used in Fig. 2.

Figure 5 depicts the condensate momentum components at the idler and signal wave number for various loss rates, comparing three different models: the many-mode GPE for the homogeneous case (1) with Eq. (B1), the corresponding three-mode model (B2), its full analytical solution (B6). The agreement is good for this homogeneous case, as expected. We find that Eq. (B8) can also provide useful guidance in the inhomogeneous multimode cases discussed in the main text.

\section{APPENDIX D: DISPERSIVE EFFECTS}

The scheme to engineer momentum-selective loss introduced in Sec. V also gives rise to an unavoidable (but small) dispersive contribution to the atomic energy, which we discuss in this Appendix.

The optical $\Lambda$ scheme causes the ground state $|g\rangle$ to be weakly dressed with the other two electronic states. Besides the desired dissipative effects discussed above, this will cause an energy shift (light shift) for the atoms, given by $\delta E(k)=$ $\operatorname{Tr}\left[\hat{\rho}^{(\infty)} \hat{H}_{\text {EIT }}\right]$ that also will depend on the atomic velocity.

We find

$$
\begin{aligned}
\delta E(k)= & -\delta \Omega_{p}^{2}\left[\frac{3}{2} \gamma^{3} \delta^{2}+\frac{1}{2} \Gamma \Omega_{c}^{2}\left(4 \Delta_{p} \delta+\Omega_{c}^{2}+\Omega_{c}^{2}\right)\right. \\
& +\frac{\Gamma}{2}\left\{4 \delta\left[\delta\left(4 \Delta_{c}^{2}+\frac{\Gamma^{2}}{2}\right)+\Omega_{c}^{2}\left(3 \Delta_{c}-2 \Delta_{p}\right)\right]\right. \\
& \left.\left.+\Omega_{p}^{2}\left(\Omega_{c}^{2}-8 \Delta_{c} \delta\right)+\Omega_{p}^{4}\right\}\right] \\
& \times\left\{\frac{\Gamma \Omega_{c}^{2}}{2}\left[4\left(\Gamma^{2}+4 \Delta_{p}^{2}\right) \delta^{2}+8 \Delta_{p} \delta \Omega_{c}^{2}+\Omega_{c}^{4}\right]\right. \\
& +\Omega_{p}^{2}\left[2 \Gamma\left(\Gamma^{2}+4 \Delta_{c}^{2}\right) \delta^{2}+8 \Gamma \delta^{2} \Omega_{c}^{2}+\frac{3}{2} \Gamma \Omega_{c}^{4}\right] \\
& \left.+\Omega_{p}^{4}\left[\frac{3}{2} \Gamma \Omega_{c}^{2}-4 \Gamma \Delta_{c} \delta\right]+\frac{1}{2} \Gamma \Omega_{p}^{6}\right\}^{-1},
\end{aligned}
$$

with $\delta=\left(\Delta_{c}-\Delta_{p}\right)$.

In comparison with the free-atom dispersion relation $E=$ $\hbar^{2} k^{2} /(2 m)$ this effect remains small but not entirely negligible, as seen in Fig. 6.

\section{APPENDIX E: MODELLING QUANTUM NOISE USING TRUNCATED WIGNER APPROXIMATION}

Following initial discussions [52-54] the TWA in a BEC context is described in many articles, including Refs. [55-57]. Very briefly, the method amounts to carefully adding random noise to the initial state of the GPE, Eq. (1), in order to provide an estimate for the effects of quantum depletion or thermal fluctuations beyond the mean-field analysis [74].

Under the assumption of only atomic contact scattering, the TWA equation of motion remains identical to the GPE. However, when dissipation is taken into account, these equations are modified [58] and a dynamical noise terms in the equation of motion appears [59]. Following Ref. [59], we 


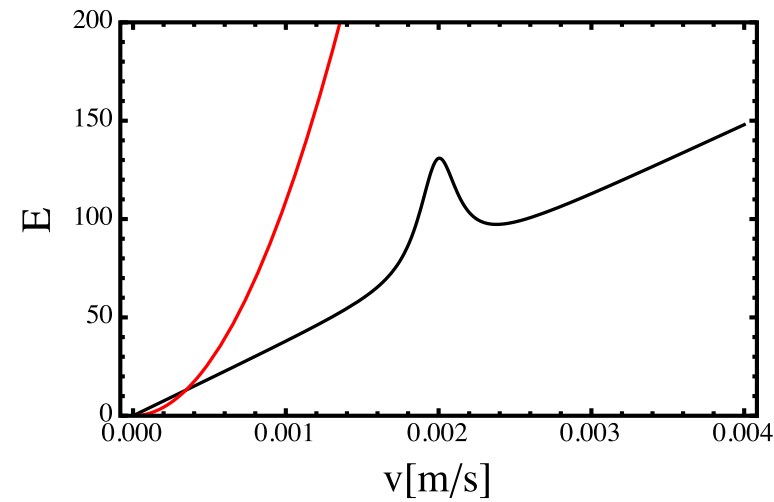

FIG. 6. (black) Light shift from laser coupling $\delta E(k)$, Eq. (D1), compared with kinetic energy $E_{\text {kin }}=\frac{1}{2} m v^{2}$ (red). Since the former is much smaller than the latter, it does not significantly affect the condensate dynamics.

obtain the stochastic differential equation (SDE):

$$
\begin{aligned}
i \hbar d \alpha(k)= & {\left[\frac{\hbar^{2} k^{2}}{2 m} \alpha(k)+U \int_{-\infty}^{\infty} d k_{1} \int_{-\infty}^{\infty} d k_{2} \int_{-\infty}^{\infty} d k_{3}\right.} \\
& \times \delta\left(k_{2}+k_{3}-k_{1}-k\right) \alpha^{*}\left(k_{1}\right) \alpha\left(k_{2}\right) \alpha\left(k_{3}\right) \\
& \left.-i \hbar \frac{\gamma(k)}{2} \alpha(k)\right] d t+i \hbar \sqrt{\frac{\gamma(k)}{2}} d \xi(k, t),
\end{aligned}
$$

where $\alpha(k)$ is the TWA stochastic field in momentum space, and $d \xi(k, t)$ are complex Gaussian noises that fulfill $\overline{d \xi(k, t) d \xi\left(k^{\prime}, t\right)}=0 \quad$ and $\quad \overline{d \xi(k, t)^{*} d \xi\left(k^{\prime}, t\right)}=\delta\left(k-k^{\prime}\right) d t$. The initial condition is $\alpha(k, t=0)=\phi(k, t=0)+\eta(k) / \sqrt{2}$, where $\phi(k, t=0)$ is the mean-field initial condition and $\eta(k)$ are again unit variance complex Gaussian noises similar to $d \xi(k, t)$.

A different symbol $\alpha(k)$ has been chosen for the stochastic field compared with the mean field $\phi(k)$, to emphasize the difference in physical interpretation due to the presence of noise. The total atomic density, for instance, is now given by $n_{\text {tot }}(k)=\overline{|\alpha(k)|^{2}}-1 /(2 \Delta k)$, where $\cdots$ denotes stochastic averaging, and $\Delta k$ is the numerical momentum grid spacing.

The noise term $\sim d \xi(k, t)$ ensures that momentum modes retain their initial noise population despite the presence of loss $\sim \gamma(k)$. In practice, we restrict all noises to the center part of the numerical momentum-space domain as in Ref. [57], to reduce aliasing.

In our numerical studies, we have averaged over 6400 and 64 trajectories for Figs. 2 and 3, respectively. Moreover, in Fig. 3 we have adjusted the noise level by scaling both $d \xi$ and $\eta$ such that the total noise density amounts to a depletion $d=8\left(\rho_{0} a_{s}\right)^{1 / 3} /(3 \sqrt{\pi})$ [75], where $\rho_{0}$ is the 3D peak density. This reflects our aim to model a quasi-2D, instead of a genuinely 2D BEC.
[1] R. W. Boyd, Nonlinear Optics (Academic Press, San Diego, 2003).

[2] S. Inouye, T. Pfau, S. Gupta, A. P. Chikkatur, A. Görlitz, D. E. Pritchard, and W. Ketterle, Nature (London) 402, 641 (1999).

[3] V. Krachmalnicoff, J.-C. Jaskula, M. Bonneau, V. Leung, G. B. Partridge, D. Boiron, C. I. Westbrook, P. Deuar, P. Ziń, M. Trippenbach et al., Phys. Rev. Lett. 104, 150402 (2010).

[4] L. Deng, E. W. Hagley, J. Wen, M. Trippenbach, Y. Band, P. S. Julienne, J. E. Simsarian, K. Helmerson, S. L. Rolston, and W. D. Phillips, Nature (London) 398, 218 (1999).

[5] M. Trippenbach, Y. B. Band, and P. S. Julienne, Phys. Rev. A 62, 023608 (2000).

[6] R. Bücker, J. Grond, S. Manz, T. Berrada, T. Betz, C. Koller, U. Hohenester, T. Schumm, A. Perrin, and J. Schmiedmayer, Nat. Phys. 7, 608 (2011).

[7] R. Bücker, U. Hohenester, T. Berrada, S. van Frank, A. Perrin, S. Manz, T. Betz, J. Grond, T. Schumm, and J. Schmiedmayer, Phys. Rev. A 86, 013638 (2012).

[8] C. Gross, H. Strobel, E. Nicklas, T. Zibold, N. Bar-Gill, G. Kurizki, and M. K. Oberthaler, Nature (London) 480, 219 (2011).

[9] K. Mølmer, New J. Phys. 8, 170 (2006).

[10] R. El-Ganainy, K. G. Makris, D. N. Christodoulides, and Z. H. Musslimani, Opt. Lett. 32, 2632 (2007).

[11] C. E. Ruter, K. Makris, R. El-Ganainy, D. Christodoulides, M. Segev, and D. Kip, Nat. Phys. 6, 192 (2010).

[12] B. Peng, S. K. Ozdemir, F. Lei, F. Monifi, M. Gianfreda, G. L. Long, S. Fan, F. Nori, C. M. Bender, and L. Yang, Nat. Phys. 10, 394 (2014).
[13] R. El-Ganainy, M. Khajavikhan, and L. Ge, Phys. Rev. A 90, 013802 (2014).

[14] R. El-Ganainy, L. Ge, M. Khajavikhan, and D. N. Christodoulides, Phys. Rev. A 92, 033818 (2015).

[15] H. Jing, S. K. Ozdemir, X.-Y. Lu, J. Zhang, L. Yang, and F. Nori, Phys. Rev. Lett. 113, 053604 (2014).

[16] D. Schönleber, A. Eisfeld, and R. El-Ganainy, New J. Phys. 18, 045014 (2016).

[17] M. Wimmer, A. Regensburger, M. Miri, C. Bersch, D. Christodoulides, and U. Peschel, Nature Commun. 6, 7782 (2015).

[18] L. Ge and R. El-Ganainy, Sci. Rep. 6, 24889 (2016).

[19] M. H. Teimourpour, L. Ge, D. N. Christodoulides, and R. ElGanainy, Sci. Rep. 6, 33253 (2016).

[20] R. El-Ganainy, J. I. Dadap, and R. M. Osgood, Opt. Lett. 40, 5086 (2015).

[21] T. Wasak, P. Szańkowski, V. V. Konotop, and M. Trippenbach, Opt. Lett. 40, 5291 (2015).

[22] D. A. Antonosyan, A. S. Solntsev, and A. A. Sukhorukov, Opt. Lett. 40, 4575 (2015).

[23] G. K. Campbell, J. Mun, M. Boyd, E. W. Streed, W. Ketterle, and D. E. Pritchard, Phys. Rev. Lett. 96, 020406 (2006).

[24] K. M. Hilligsøe and K. Mølmer, Phys. Rev. A 71, 041602 (2005).

[25] C. Klempt, O. Topic, G. Gebreyesus, M. Scherer, T. Henninger, P. Hyllus, W. Ertmer, L. Santos, and J. J. Arlt, Phys. Rev. Lett. 104, 195303 (2010).

[26] G. Morigi, J. Eschner, and C. H. Keitel, Phys. Rev. Lett. 85, 4458 (2000).

[27] G. Morigi, Phys. Rev. A 67, 033402 (2003). 
[28] C. F. Roos, D. Leibfried, A. Mundt, F. Schmidt-Kaler, J. Eschner, and R. Blatt, Phys. Rev. Lett. 85, 5547 (2000).

[29] M. Fleischhauer, A. Imamoglu, and J. P. Marangos, Rev. Mod. Phys. 77, 633 (2005).

[30] A. K. Mohapatra, T. R. Jackson, and C. S. Adams, Phys. Rev. Lett. 98, 113003 (2007).

[31] A. K. Mohapatra, M. G. Bason, B. Butscher, K. J. Weatherill, and C. S. Adams, Nat. Phys. 4, 890 (2008).

[32] S. Mauger, J. Millen, and M. P. A. Jones, J. Phys. B: At., Mol. Opt. Phys. 40, F319 (2007).

[33] S. S. Hodgman, R. G. Dall, L. J. Byron, K. G. H. Baldwin, S. J. Buckman, and A. G. Truscott, Phys. Rev. Lett. 103, 053002 (2009).

[34] V. V. Konotop, J. Yang, and D. A. Zezyulin, Rev. Mod. Phys. 88, 035002 (2016).

[35] S. V. Suchkov, A. A. Sukhorukov, J. Huang, S. V. Dmitriev, C. Lee, and Y. S. Kivshar, Laser Photonics Rev. 10, 177 (2016).

[36] S. A. Haine and A. J. Ferris, Phys. Rev. A 84, 043624 (2011).

[37] R. G. Dall, L. J. Byron, A. G. Truscott, G. R. Dennis, M. T. Johnsson, and J. J. Hope, Phys. Rev. A 79, 011601(R) (2009).

[38] T. Wasak, V. V. Konotop, and M. Trippenbach, Phys. Rev. A 88, 063626 (2013).

[39] A. Perrin, C. M. Savage, D. Boiron, V. Krachmalnicoff, C. I. Westbrook, and K. V. Kheruntsyan, New J. Phys. 10, 045021 (2008).

[40] A. J. Ferris, M. K. Olsen, and M. J. Davis, Phys. Rev. A 79, 043634 (2009).

[41] A. Perrin, H. Chang, V. Krachmalnicoff, M. Schellekens, D. Boiron, A. Aspect, and C. I. Westbrook, Phys. Rev. Lett. 99, 150405 (2007).

[42] J.-C. Jaskula, M. Bonneau, G. B. Partridge, V. Krachmalnicoff, P. Deuar, K. V. Kheruntsyan, A. Aspect, D. Boiron, and C. I. Westbrook, Phys. Rev. Lett. 105, 190402 (2010).

[43] F. Dalfovo, S. Giorgini, L. P. Pitaevskii, and S. Stringari, Rev. Mod. Phys. 71, 463 (1999).

[44] P. J. Y. Louis, E. A. Ostrovskaya, C. M. Savage, and Y. S. Kivshar, Phys. Rev. A 67, 013602 (2003).

[45] B. Eiermann, T. Anker, M. Albiez, M. Taglieber, P. Treutlein, K.-P. Marzlin, and M. K. Oberthaler, Phys. Rev. Lett. 92, 230401 (2004).

[46] T. P. Meyrath, F. Schreck, J. L. Hanssen, C.-S. Chuu, and M. G. Raizen, Phys. Rev. A 71, 041604 (2005).

[47] Online at www.xmds.org

[48] G. R. Dennis, J. J. Hope, and M. T. Johnsson, Comput. Phys. Commun. 184, 201 (2013).

[49] We extract the initial signal population $n_{s}$ from the full nonhomogeneous simulation and choose a spatial domain of length $L_{s}$ for the homogeneous case that matches the support of the signal wave packet $L_{s} \approx \sigma$.

[50] I. I. Beterov, I. I. Ryabtsev, D. B. Tretyakov, and V. M. Entin, Phys. Rev. A 79, 052504 (2009).
[51] See Supplemental Material at http://link.aps.org/supplemental/ 10.1103/PhysRevA.96.013605 for additional movies showing the dynamics presented in Figs. 2 and 3.

[52] M. J. Steel, M. K. Olsen, L. I. Plimak, P. D. Drummond, S. M. Tan, M. J. Collett, D. F. Walls, and R. Graham, Phys. Rev. A 58, 4824 (1998).

[53] A. Sinatra, C. Lobo, and Y. Castin, Phys. Rev. Lett. 87, 210404 (2001).

[54] A. Sinatra, C. Lobo, and Y. Castin, J. Phys. B: At., Mol. Opt. Phys. 35, 3599 (2002).

[55] S. Wüster, B. J. Dąbrowska-Wüster, A. S. Bradley, M. J. Davis, P. B. Blakie, J. J. Hope, and C. M. Savage, Phys. Rev. A 75, 043611 (2007).

[56] S. Wüster, B. J. Dąbrowska-Wüster, S. M. Scott, J. D. Close, and C. M. Savage, Phys. Rev. A 77, 023619 (2008).

[57] B. J. Dąbrowska-Wüster, S. Wüster, and M. J. Davis, New J. Phys. 11, 053017 (2009).

[58] J. Dziarmaga and K. Sacha, Phys. Rev. A 68, 043607 (2003).

[59] A. A. Norrie, R. J. Ballagh, C. W. Gardiner, and A. S. Bradley, Phys. Rev. A 73, 043618 (2006).

[60] N. R. Thomas, N. Kjærgaard, P. S. Julienne, and A. C. Wilson, Phys. Rev. Lett. 93, 173201 (2004).

[61] C. Buggle, J. Léonard, W. von Klitzing, and J. T. M. Walraven, Phys. Rev. Lett. 93, 173202 (2004).

[62] J. Kofler, M. Singh, M. Ebner, M. Keller, M. Kotyrba, and A. Zeilinger, Phys. Rev. A 86, 032115 (2012).

[63] M. Ögren and K. V. Kheruntsyan, Phys. Rev. A 79, 021606 (2009).

[64] J. B. Balewski, A. T. Krupp, A. Gaj, D. Peter, H. P. Büchler, R. Löw, S. Hofferberth, and T. Pfau, Nature (London) 502, 664 (2013).

[65] T. Karpiuk, M. Brewczyk, K. Rzążewski, J. B. Balewski, A. T. Krupp, A. Gaj, R. Löw, S. Hofferberth, and T. Pfau, New J. Phys. 17, 053046 (2015).

[66] N. Henkel, R. Nath, and T. Pohl, Phys. Rev. Lett. 104, 195302 (2010).

[67] S. Möbius, M. Genkin, A. Eisfeld, S. Wüster, and J.-M. Rost, Phys. Rev. A 87, 051602(R) (2013).

[68] R. Mukherjee, C. Ates, W. Li, and S. Wüster, Phys. Rev. Lett. 115, 040401 (2015).

[69] K. Leonhardt, S. Wüster, and J. M. Rost, J. Phys. B: At., Mol. Opt. Phys. 50, 054001 (2017).

[70] J. Wang, M. Gacesa, and R. Côté, Phys. Rev. Lett. 114, 243003 (2015).

[71] E. A. Donley, N. R. Claussen, S. L. Cornish, J. L. Roberts, E. A. Cornell, and C. E. Wieman, Nature (London) 412, 295 (2001).

[72] S. Wüster, J. J. Hope, and C. M. Savage, Phys. Rev. A 71, 033604 (2005).

[73] S. Wüster, Phys. Rev. A 78, 021601(R) (2008).

[74] A. A. Norrie, R. J. Ballagh, and C. W. Gardiner, Phys. Rev. A 73, 043617 (2006).

[75] C. J. Pethik and H. Smith, Bose-Einstein Condensation in Dilute Gases (Cambridge University Press, Cambridge, England, 2002). 